\title{
Optimal periodic control of spin systems: Application to the maximization of the signal to noise ratio per unit time
}

\author{
N. Jbili ${ }^{7,8}$, K. Hamraoui ${ }^{2}$, S. J. Glaser ${ }^{1}$, J. Salomon ${ }^{4,5,6}$, and D. Sugny ${ }^{2,3 *}$ \\ ${ }^{1}$ Department of Chemistry, Technische Universität München, \\ Lichtenbergstrasse 4, D-85747 Garching, Germany \\ 2 Laboratoire Interdisciplinaire Carnot de Bourgogne (ICB), \\ UMR 6303 CNRS-Université de Bourgogne-Franche Comté, \\ 9 Av. A. Savary, BP 47 870, F-21078 DIJON Cedex, FRANCE \\ 3 Institute for Advanced Study, Technische Universität München, \\ Lichtenbergstrasse 2 a, D-85748 Garching, Germany \\ 4 INRIA Paris, ANGE Project-Team, 75589 Paris Cedex 12, France \\ 5 Sorbonne Universits, UPMC Univ Paris 06, UMR 7598, \\ Laboratoire Jacques-Louis Lions, F-75005, Paris, France \\ ${ }^{6}$ CNRS, UMR 7598, Laboratoire Jacques-Louis Lions, F-75005, Paris \\ 7 Paris Dauphine University, PSL Research University, CNRS, CEREMADE, 75016 Paris, France and \\ ${ }^{8}$ University of Sousse, High School of Sciences and Technology, LAMMDA, 4011 Hammam Sousse, Tunisia
}

(Dated: January 6, 2019)

\begin{abstract}
We propose an optimal control algorithm for periodic spin dynamics. This non-trivial optimization problem involves the design of a control field maximizing a figure of merit, while finding the initial and final states of the dynamics, which are not known but are subjected to specific periodic conditions. As an illustrative example, we consider the maximization of the signal to noise ratio per unit time of spin systems. In the case of a homogeneous spin ensemble and a very short control duration, we show numerically that the optimal field corresponds to the Ernst angle solution. We investigate the optimal control process for longer control durations and their sensitivity to offset inhomogeneities.
\end{abstract}

\section{INTRODUCTION}

Optimality with respect to a given criterion is vital in many applications, but it presents a complexity that requires a lot of ingenuity to provide a solution [1-4]. Quantum control [5-11] is no exception to this rule and many efforts have been made recently in this domain to develop tools and methods in order to conduct a systematic analysis of optimal control problems (see, e.g., some recent reviews [12-15] and references therein). In quantum control, there is a genuine desire to solve concrete questions and contribute beyond the purely theoretical analysis $[12,13]$. Different numerical iterative algorithms have been proposed to solve the optimal equations $[16-21]$ in a variety of domains $[12,13]$ extending from photochemistry [5, 6], Nuclear Magnetic Resonance (NMR) [22-24] and Magnetic Resonance Imaging (MRI) [25-34], and in quantum information science [12]. Several modifications of standard algorithms have been proposed to account for experimental limitations and uncertainties [17, 22-24, 35-41], showing their flexibility and the possibility to adapt them to new classes of control problems.

We propose in this work to investigate a new issue in quantum control, namely the optimal control of periodic processes. The originality and the difficulty of this optimization problem come from the fact that the

\footnotetext{
*Electronic address: dominique.sugny@u-bourgogne.fr
}

initial and final states of the dynamical system are not known, but have to be determined together with the control field to maximize a figure of merit. The optimal control of periodic processes is well-established in mathematics $[42,43]$ and have been applied in different domains, such as robotics or biology [44], to mention a few. To the best of our knowledge, this issue has not been explored in quantum control. As an illustrative example, we consider in this paper a question of fundamental and practical interest in NMR and MRI [45-48], namely the maximization of the signal-to-noise ratio per unit time (SNR) of spin $1 / 2$ particles. The SNR is practically enhanced in spin systems by using a multitude of identical cycles. In this periodic regime, the SNR increases as the square root of the number of scans. Each elementary block is composed of a detection time and of a control period where the spin is subjected to a radio-frequency magnetic pulse, this latter being used to guarantee the periodic character of the overall process. A first solution to this problem was established in the sixties by R. Ernst and his co-workers [48]. In this protocol, the control law is made of a $\delta$ - pulse, characterized by a specific rotation angle, called the Ernst angle solution. This pulse sequence is nowadays currently used in magnetic resonance spectroscopy and imaging. Related control procedures, known as SSFP (Steady State Free Precession) have been also intensively investigated in the literature for medical applications (See Ref. [49-56], to mention a few). Some of us have revisited recently the question of the Ernst angle procedure by applying the tools of geometric control theory $[3,4]$. In [57], it is shown in the general case of unbounded controls which also includes 
finite-amplitude shaped pulses (only $\delta$ - pulses were considered in [48]) that the Ernst angle solution is the optimal solution of the control problem aiming at maximizing the SNR of a spin. This analysis was generalized in [58] to spin dynamics in presence of radiation damping effects and crusher gradients.

The maximization of the SNR is used in this work as a motivation to extend the scope of quantum optimal algorithms to periodic dynamics. The periodicity constraint is enforced through the introduction of a Lagrange multiplier. We outline the principles of a gradient optimization procedure in this generalized framework. We investigate numerically the optimization of the SNR. For short control times, we show that the algorithm converges to the Ernst angle solution in the case of a homogeneous spin ensemble. For longer control durations, we observe that the optimal solution heavily depends on the guess field used to initialize the algorithm. We describe geometrically the different solutions. Finally, we consider the case of an inhomogeneous ensemble of spins with several offsets. For a fixed control time, we analyze the different periodic trajectories and the maximum achievable SNR.

The paper is organized as follows. Section II introduces the model system and gives a complete description of the optimal control problem. Section III outlines the principles of the new optimization procedure. A special attention is paid to the difference with a standard iterative algorithm. Section IV is dedicated to the numerical implementation of the algorithm. The numerical results are presented and discussed in Sec. $\mathrm{V}$ for homogeneous and inhomogeneous spin ensembles, respectively. Conclusion and prospective views are given in Sec. VI. Some analytical computations about the Ernst angle solution are reported in Appendix A.

\section{DESCRIPTION OF THE CONTROL PROCESS FOR THE MAXIMIZATION OF THE SNR}

We consider an inhomogeneous ensemble of uncoupled spin $1 / 2$ particles with different offset terms [47]. In a given rotating frame, the equation of motion for the spin ensemble reads:

$$
\begin{aligned}
\left(\begin{array}{c}
\dot{x}^{(\omega)} \\
\dot{y}^{(\omega)} \\
\dot{z}^{(\omega)}
\end{array}\right) & =\left(\begin{array}{c}
-2 \pi x^{(\omega)} / T_{2} \\
-2 \pi y^{(\omega)} / T_{2} \\
2 \pi\left(1-z^{(\omega)}\right) / T_{1}
\end{array}\right)+ \\
& \left(\begin{array}{c}
-\omega y^{(\omega)}+\omega_{y}(t) z^{(\omega)} \\
\omega x^{(\omega)}-\omega_{x}(t) z^{(\omega)} \\
\omega_{x}(t) y^{(\omega)}-\omega_{y}(t) x^{(\omega)}
\end{array}\right),
\end{aligned}
$$

where the Bloch vector $X:=\left(x^{(\omega)}, y^{(\omega)}, z^{(\omega)}\right)^{\top}$ represents the state of an element of the ensemble, $T_{1}$ and $T_{2}$ are the two relaxation parameters, $\omega$ the offset term and $\omega_{x}(t)$, $\omega_{y}(t)$ the two control fields. We use normalized coordinates so that the Bloch ball is defined by $x^{2}+y^{2}+z^{2} \leq 1$ for each spin. Normalizing the time by the detection time
$T_{d}$ (see below for the definition) and setting $\gamma=2 \pi T_{d} / T_{1}$ and $\Gamma=2 \pi T_{d} / T_{2}$, we arrive at:

$$
\dot{X}=A(\vec{\omega}) X+D
$$

where $D:=(0,0, \gamma)^{\top}$ and $A(\vec{\omega})$ is a $3 \times 3$ - matrix:

$$
A(\vec{\omega}):=\left(\begin{array}{ccc}
-\Gamma & -\omega & \omega_{y}(t) \\
\omega & -\Gamma & -\omega_{x}(t) \\
-\omega_{y}(t) & \omega_{x}(t) & -\gamma
\end{array}\right)
$$

with $\vec{\omega}(t)=\left(\omega_{x}(t), \omega_{y}(t)\right)$. The optimization of the SNR per unit time is described by a simple scenario (see the schematic description in Fig. 1 and Ref. [57, 58] for details). The point $M^{(\omega)}$ reached at the end of the control process is the measurement point for the spin of offset $\omega$. The corresponding spin has then a free evolution from this point to the steady state $S^{(\omega)}$ where the pulse sequence starts. The times $T_{d}$ and $T_{c}$ denote the detection time (fixed by the experimental setup) and the control time, respectively. The total time during which a series of

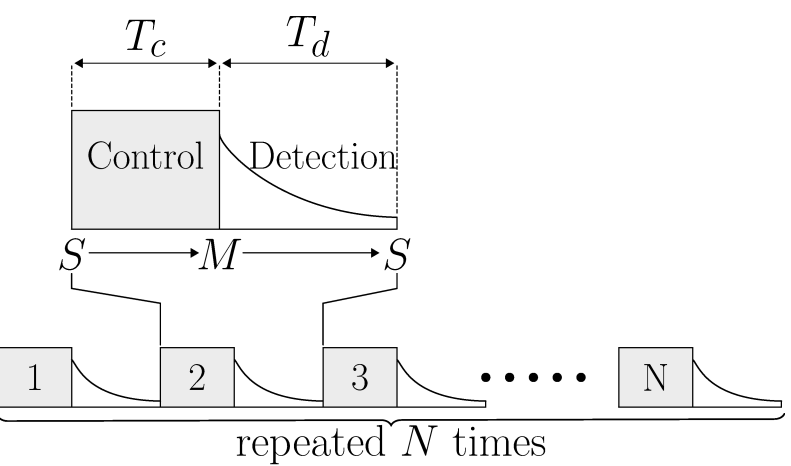

FIG. 1: Schematic representation of the cyclic process used in the maximization of the SNR.

identical experiments are made is fixed. The total number $N$ of experiments is then given by $T=N\left(T_{c}+T_{d}\right)$. The optimization problem is defined through the introduction of a figure of merit:

$$
R=\frac{N}{\sqrt{N}} \sqrt{\left[\sum_{\omega} x^{(\omega)}\left(T_{c}\right)\right]^{2}+\left[\sum_{\omega} y^{(\omega)}\left(T_{c}\right)\right]^{2}},
$$

where $\left[\sum_{\omega} x^{(\omega)}\left(T_{c}\right)\right]^{2}+\left[\sum_{\omega} y^{(\omega)}\left(T_{c}\right)\right]^{2}$ is the square modulus of the strength of the signal (transverse magnetization) at time $T_{c}$. We consider a white noise, which leads to the $\sqrt{N}$ factor in $R$. Using the relation $N=$ $T /\left(T_{c}+T_{d}\right)$ and setting $T_{d}=1$, we define the normalized figure of merit:

$$
F_{\mathrm{opt}}=\frac{1}{\sqrt{1+T_{c}}} \sqrt{\left[\sum_{\omega} x^{(\omega)}\left(T_{c}\right)\right]^{2}+\left[\sum_{\omega} y^{(\omega)}\left(T_{c}\right)\right]^{2}} .
$$

This figure of merit $F_{\text {opt }}$ will be used in the numerical simulations of Sec. V. 


\section{OPTIMAL PERIODIC CONTROL OF SPIN SYSTEMS}

\section{A. Optimal control algorithm}

In this paragraph, we generalize the optimal control algorithm GRAPE to periodic dynamics [16]. To simplify the presentation of the optimization procedure, we specifically consider the case of the maximization of the SNR of a single spin, but the algorithm can be applied to any periodic control of quantum systems. We focus here on the general characteristics of the algorithm, a numerical implementation is described in Sec. IV.

We describe the dynamics of the model system on bounded intervals ( $\left.\left[\begin{array}{ll}0 & T_{c}\end{array}\right],\left[T_{c} T_{c}+T_{d}\right], \cdots\right)$, keeping in mind that all the variables are $\left(T_{c}+T_{d}\right)$ - periodic. Since the dynamics are governed by two different regimes over a period, we split the propagation into the intervals $\left[0, T_{c}\right]$ and $\left[T_{c}, T_{c}+T_{d}\right]$ :

$$
\begin{cases}X\left(T_{c}\right) & =U_{c} X(0)+E_{c} \\ X(0) & =U_{d} X\left(T_{c}\right)+E_{d}\end{cases}
$$

where the indices $c$ and $d$ stand respectively for the controlled and detection periods, $U_{c}$ and $U_{d}$ (resp. $E_{c}$ and $E_{d}$ ) denote the linear (resp. affine) parts of the propagators over the intervals $\left[0, T_{c}\right]$ and $\left[T_{c}, T_{c}+T_{d}\right]$. Setting $W:=U_{c} U_{d}$ and $L:=U_{c} E_{d}+E_{c}$, we find that the periodicity constraint on $X$ reads:

$$
X\left(T_{c}\right)=W X\left(T_{c}\right)+L
$$

that is

$$
X\left(T_{c}\right)=(\mathbf{1}-W)^{-1} L,
$$

in the case where $\mathbf{1}-W$ is invertible, 1 being the $3 \times 3$ identity matrix. The invertibility of $\mathbf{1}-W$, which is connected to the existence of periodic trajectories, is examined in Sec. III B.

The optimal control problem is defined through the figure of merit $F\left(X\left(T_{c}\right)\right)=\left(1+T_{c}\right) F_{\text {opt }}^{2}$. To take into account the different constraints on the dynamics, we use the method of Lagrange multipliers by introducing the Lagrangian of the problem:

$$
\begin{aligned}
\tilde{F}= & F\left(X\left(T_{c}\right)\right) \\
& +\int_{T_{c}}^{2 T_{c}+T_{d}} Y(t)^{\top}(A(\vec{\omega}) X(t)+D-\dot{X}(t)) d t,
\end{aligned}
$$

where $Y(t)$ is the $\left(T_{c}+T_{d}\right)$ - periodic adjoint state of the system at time $t$. Since $F$ explicitly depends on $X\left(T_{c}\right)$, $Y$ has a jump at time $T_{c}$, i.e. $Y\left(T_{c}^{-}\right) \neq Y\left(T_{c}^{+}\right)$. Differentiating $\tilde{F}$ with respect to its variables, we deduce the necessary conditions for $\vec{\omega}$ to be optimal, namely:

$$
\left\{\begin{array}{l}
F^{\prime}\left(X\left(T_{c}\right)\right)-\left(Y\left(T_{c}^{-}\right)-Y\left(T_{c}^{+}\right)\right)=0 \\
\dot{Y}(t)+A(\vec{\omega})^{\top} Y(t)=0 \\
Y^{\top} \partial_{\vec{\omega}} A(\vec{\omega}) X=0
\end{array}\right.
$$

completed by Eq. (1), where $F^{\prime}\left(X\left(T_{c}\right)\right)=\nabla_{X\left(T_{c}\right)} F$. Using the fact that $Y\left(T_{c}^{+}\right)=U_{d}^{\top} Y\left(T_{c}+T_{d}^{-}\right)$and $Y\left(T_{c}+\right.$ $\left.T_{d}^{+}\right)=U_{c}^{\top} Y\left(T_{c}^{-}\right)$, we then obtain:

$$
Y\left(T_{c}^{+}\right)=U_{d}^{\top} U_{c}^{\top} Y\left(T_{c}^{-}\right)=W^{\top} Y\left(T_{c}^{-}\right) .
$$

Combining the latter with Eq. (8), we get:

$$
\left(\mathbf{1}-W^{\top}\right) Y\left(T_{c}^{-}\right)=F^{\prime}\left(X\left(T_{c}\right)\right) .
$$

If $\mathbf{1}-W^{\boldsymbol{\top}}$ is invertible, we deduce that the condition at time $t=T_{c}^{-}$for the adjoint state is:

$$
Y\left(T_{c}^{-}\right)=\left(\mathbf{1}-W^{\boldsymbol{\top}}\right)^{-1} F^{\prime}\left(X\left(T_{c}\right)\right) .
$$

The optimality system (1) and (8) can be solved by using Eq. (6) and (9) and the following iterative approach similar in spirit to a standard gradient algorithm. The iteration is initialized by a guess field $\vec{\omega}_{0}(t)$. At each step, $X\left(T_{c}\right)$ is computed using Eq. (6) which then enables to find a solution of Eq. (1). Then we deduce the state $Y\left(T_{c}^{-}\right)$from Eq. (9) which allows to compute the solution of Eq. (8). The correction to the control field is finally determined using $\nabla_{\vec{\omega}_{k}} \tilde{F}$. The detailed numerical procedure is described in Sec. IV in a time-discretized setting.

\section{B. Proof of the existence of periodic trajectories}

We present a proof of the existence of periodic trajectories for any control field $\vec{\omega}(t)$ and any offset $\omega$. We start by writing the Bloch equation (1) as follows:

$$
\dot{X}(t)=(B+C(t)) X(t)+D
$$

where

$$
B:=\left(\begin{array}{ccc}
-\Gamma & -\omega & 0 \\
\omega & -\Gamma & 0 \\
0 & 0 & -\gamma
\end{array}\right)
$$

and

$$
C(t):=\left(\begin{array}{ccc}
0 & 0 & \omega_{y}(t) \\
0 & 0 & -\omega_{x}(t) \\
-\omega_{y}(t) & \omega_{x}(t) & 0
\end{array}\right)
$$

We denote by $P(t)$ the solution of Eq. (10) when $D=0$ :

$$
\dot{P}(t)=(B+C(t)) P(t) .
$$

Since the matrix $B$ in Eq. (1) is skew-symmetric, the norm of $P(t)$ decreases when $t$ increases. Indeed, we have:

$$
\frac{d\|P(t)\|^{2}}{d t}=-2\left(\Gamma x^{2}(t)+\Gamma y^{2}(t)+\gamma z^{2}(t)\right)<0 .
$$

As a consequence, for $t \in[0, T]$, we have

$$
\frac{d\|P(t)\|^{2}}{d t} \leq-2 \min (\Gamma, \gamma)\|P(t)\|^{2}
$$


Defining $g(t):=\frac{d\|P(t)\|^{2}}{d t}+2 \min (\Gamma, \gamma)\|P(t)\|^{2}$ and multiplying both sides by $e^{2 \min (\Gamma, \gamma) t}$, we obtain:

$$
\frac{d\left(e^{2 \min (\Gamma, \gamma) t}\|P(t)\|^{2}\right)}{d t}=e^{2 \min (\Gamma, \gamma) t} g(t) .
$$

The solution of Eq. (11) can be expressed as:

$\|P(t)\|^{2}=e^{-2 \min (\Gamma, \gamma) t}\|P(0)\|^{2}+\int_{0}^{t} e^{2 \min (\Gamma, \gamma)(s-t)} g(s) d s$.

Since $g(t) \leq 0$, it follows that:

$$
\|P(t)\| \leq e^{-\min (\Gamma, \gamma) t}\|P(0)\| .
$$

The inequality (12) is used in the proof of the following lemma.

Lemma 1. Let the function $f$ be defined by:

$$
\begin{aligned}
& f(X(0)):=e^{T B} X(0)+\int_{0}^{T_{c}} e^{(T-s) B}(C(s) X(s)+D) d s \\
& +\int_{T_{c}}^{T} e^{(T-s) B} D d s,
\end{aligned}
$$

where $X$ is the solution of Eq. (10). The function $f$ has a unique fixed point.

Proof Let $X$ and $X^{\prime}$ be the solutions of Eq. (10) corresponding respectively to initial conditions $X(0)$ and $X^{\prime}(0)$. Integrating Eq. (10) over a period $T=T_{c}+T_{d}$, we obtain that $X$ satisfies:

$$
\begin{aligned}
& X(T)=e^{T B} X(0)+\int_{0}^{T_{c}} e^{(T-s) B}(C(s) X(s)+D) d s \\
& +\int_{T_{c}}^{T} e^{(T-s) B} D d s,
\end{aligned}
$$

and the same for $X^{\prime}$. Subtracting the two identities, we get:

$$
\begin{aligned}
& X(T)-X^{\prime}(T)=e^{T B}\left(X(0)-X^{\prime}(0)\right)+ \\
& \int_{0}^{T_{c}} e^{(T-s) B} C(s)\left(X(s)-X^{\prime}(s)\right) d s .
\end{aligned}
$$

Setting $P(t)=X(t)-X^{\prime}(t)$, we obtain:

$$
P(T)=e^{T B} P(0)+\int_{0}^{T_{c}} e^{(T-s) B} C(s) P(s) d s,
$$

and it can be easily checked that $P$ satisfies inequality (12) on one hand, and $P(T)=f(X(0))-f\left(X^{\prime}(0)\right)$ on the other hand. It follows that

$$
\left\|f(X(0))-f\left(X^{\prime}(0)\right)\right\| \leq e^{-\min (\Gamma, \gamma) t}\|P(0)\|,
$$

which leads to

$$
\left\|f(X(0))-f\left(X^{\prime}(0)\right)\right\| \leq e^{-\min (\Gamma, \gamma) t}\left\|X(0)-X^{\prime}(0)\right\| .
$$

Since $e^{-\min (\Gamma, \gamma) t}<1$, we obtain that $f$ is a contraction mapping, which implies that $f$ admits a unique fixed point.
As a consequence of this result, we observe that the periodic dynamical model introduced in this study is wellposed in a very general mathematical setting, which includes the case of an ensemble of inhomogeneous spins.

\section{DISCRETE COMPUTATION}

We now repeat the previous computation in a timediscretized setting. This corresponds to a standard experimental framework in NMR and MRI where the used magnetic fields are piecewise constant fields [46, 47]. We introduce the time discretization parameters $K$ and $d T$ satisfying $K d T=T_{c}$ and a subdivision of the time interval $\left[0, T_{c}\right]$ given by the sequence $\left(t_{k}\right)_{k=1, \cdots, K+1}$, $t_{k}=k d T$. Equation (1) is discretized using a CrankNicholson scheme, corresponding to the iteration:

$$
\frac{X_{k+1}-X_{k}}{d T}=A\left(\vec{\omega}_{k}\right) \frac{X_{k+1}+X_{k}}{2}+D, k=1, \cdots, K .
$$

where $X_{k}, A\left(\vec{\omega}_{k}\right)$ and $\vec{\omega}_{k}$ stand respectively for $X(k d T)$, $A(\vec{\omega}(k d T))$ and $\vec{\omega}(k d T)$. Introducing the matrices $B_{k}:=$ $\mathbf{1}-\frac{d T}{2} A\left(\overrightarrow{\omega_{k}}\right), \tilde{B}_{k}:=\mathbf{1}+\frac{d T}{2} A\left(\overrightarrow{\omega_{k}}\right), U_{k}:=B_{k}^{-1} \tilde{B}_{k}$ and the vector $E_{k}:=d T B_{k}^{-1} D$, Eq. (13) becomes:

$$
X_{k+1}=U_{k} X_{k}+E_{k} \text {. }
$$

For a given control field $\vec{\omega}(t)$ acting on $\left[0, T_{c}\right]$ and the initial state $X_{1}$, we obtain:

$$
\begin{aligned}
X_{k+1}= & U_{k} U_{k-1} \cdots U_{1} X_{1} \\
& +\sum_{n=1}^{k-1}\left(\prod_{j=n+1}^{k} U_{j}\right) E_{n}+E_{k} .
\end{aligned}
$$

Note that, up to a diagonalization, $U_{d}$ and $E_{d}$ can be exactly computed. The diagonalization of $A(0)$ gives $A(0)=P D_{1} P^{\top}$, which leads to $U_{d}=P \exp \left(T_{d} D_{1}\right) P^{\top}$ and $E_{c}=P \exp \left(\left(T_{c}+T_{d} s\right) D_{1} P^{\top}\right) D d s$. We therefore keep them at the discrete level, and the discrete expressions of Eq. (4) are given by:

$$
\begin{cases}X_{K+1} & =U_{c}^{d T} X_{1}+E_{c}^{d T} \\ X_{1} & =U_{d} X_{K+1}+E_{d}\end{cases}
$$

where $U_{c}^{d T}$ and $E_{c}^{d T}$ can be expressed as:

$$
\left\{\begin{array}{l}
U_{c}^{d T}:=U_{K} U_{K-1} \cdots U_{1} \\
E_{c}^{d T}:=\sum_{k=1}^{K-1}\left(\prod_{j=k+1}^{K} U_{j}\right) E_{k}+E_{K}
\end{array}\right.
$$

Introducing $W^{d T}:=U_{c}^{d T} U_{d}$ and $L^{d T}:=U_{c}^{d T} E_{d}+E_{c}^{d T}$, we deduce that Eq. (16) lead to

$$
X_{K+1}=W^{d T} X_{K+1}+L^{d T} .
$$


It follows that a solution of Eq. (18) can be written as:

$$
X_{K+1}=\left(\mathbf{1}-W^{d T}\right)^{-1} L^{d T},
$$

which is a time-discretized version of Eq. (5). In this setting, the Lagrangian can be expressed as:

$$
\tilde{F}^{d T}=F\left(X_{K+1}\right)-\sum_{k=K}^{2 K-1} Y_{k+1}^{\top}\left(X_{k+1}-U_{k} X_{k}-E_{k}\right) .
$$

The necessary conditions for $\vec{\omega}$ to be optimal are given by Eq. (14), as well as by:

$$
F^{\prime}\left(X_{K+1}\right)-Y_{K+1}+U_{K+1}^{\top} Y_{K+2}=0
$$

and, for $k=1, \cdots, K$ :

$$
\left\{\begin{array}{l}
Y_{k}-U_{k}^{\top} Y_{k+1}=0 \\
\nabla_{\vec{\omega}_{k}} F_{d T}=0
\end{array}\right.
$$

with

$$
\nabla_{\vec{\omega}_{k}} F_{d T}=Y_{k+1}^{\top} \partial_{\vec{\omega}_{k}} U_{k} X_{k}
$$

Note that $Y_{K+1}$ and $U_{K+1}^{\top} Y_{K+2}$ correspond respectively to $Y\left(T_{c}^{-}\right)$and $Y\left(T_{c}^{+}\right)$in the continuous setting. As $Y_{1}=$ $\left(U_{c}^{d T}\right)^{\top} Y_{K+1}$ and $U_{K+1}^{\top} Y_{K+2}=U_{d}^{\top} Y_{1}$, we deduce:

$$
U_{K+1}^{\top} Y_{K+2}=U_{d}^{\top}\left(U_{c}^{d T}\right)^{\top} Y_{K+1}=\left(W^{d T}\right)^{\top} Y_{K+1} .
$$

Combining the latter with Eq. (21) leads to

$$
\left(\mathbf{1}-\left(W^{d T}\right)^{\top}\right) Y_{K+1}=F^{\prime}\left(X_{K+1}\right) .
$$

Assuming once again that $\mathbf{1}-\left(W^{d T}\right)^{\top}$ is invertible (the invertibility can be shown by adapting the reasoning presented in Sec. III B to the discrete setting), we obtain $Y_{K+1}$ from:

$$
Y_{K+1}=\left(\mathbf{1}-\left(W^{d T}\right)^{\top}\right)^{-1} F^{\prime}\left(X_{K+1}\right) .
$$

which corresponds to a discrete version of Eq. (9). Using Eq. (23), we propose the following gradient-type algorithm, that solves iteratively Eq. (22).

Algorithm: Given $\vec{\omega}^{(0)}$ the initial control field, we calculate the operator $W^{d T}=U_{K} U_{K-1} \cdots U_{1} U_{d}$. Let us assume that $\vec{\omega}^{(\ell)}$ is known at iteration $\ell$, the control $\vec{\omega}^{(\ell+1)}$ is computed by the following procedure:

1. Compute $X_{K+1}^{(\ell)}$ by Eq. (19).

2. Compute $Y_{K+1}^{(\ell)}$ according to Eq. (24).

3. Propagate forward $X^{(\ell)}$ using Eq. (14) and $X_{K+1}^{(\ell)}$.

4. Propagate backward $Y^{(\ell)}$ from $Y_{K+1}^{(\ell)}$ using Eq. (22).

5. Compute the gradient $\nabla_{\vec{\omega}^{(\ell)}} F_{d T}$ according to Eq. (23), and the optimal ascent step $\rho$. Then, update the control field as follows:

$$
\vec{\omega}^{(\ell+1)}=\vec{\omega}^{(\ell)}+\rho \nabla_{\vec{\omega}^{(\ell)}} F_{d T} .
$$

Numerical results are presented in Sec. IV based on the implementation of the Crank-Nicholson approach (14). In these numerical tests, different values of $T_{c}$ are used. In order to control the accuracy of the numerical evolution of the trajectory of the state $\vec{X}$, we consider an adaptive time step in which the parameters $d T$ and $K$ are fixed with respect to the maximum amplitude of the control field $\vec{\omega}^{*}(t)$. In this way, we define

$$
d T=\frac{\Delta T}{\max _{t}\left|\vec{\omega}^{*}(t)\right|},
$$

and

$$
K=\frac{T_{c}+d T}{d T}
$$

Step 1 corresponds to the implementation of this discretization technique.

The control is updated in Step 5 where an optimal step gradient iteration is used. One could alternatively consider other optimization methods (optimized gradient method or pseudo-Newton methods, conjugate gradient method,.. ). In the examples discussed below, the choice of a gradient method with optimal step proved to be the most efficient from a numerical point of view, in terms of number of iterations and computational time.

\section{NUMERICAL RESULTS}

\section{A. Control of a homogeneous ensemble}

This section is dedicated to the numerical maximization of the SNR in the case of a homogeneous spin ensemble.

The Ernst angle solution is the time-optimal solution maximizing the SNR $[48,57]$. In this paragraph, we use this control problem as a benchmark to evaluate the efficiency of the optimization algorithm. Without loss of generality, we can assume that the offset term is zero and that $\omega_{y}(t)=0$, the spin trajectory belonging to the $(y-z)$ - plane. We first recall the definition of the Ernst angle solution. For sake of completeness, the derivation of this control protocol is given in Appendix A. In this approach, the pulse sequence is only made of a $\delta$-pulse characterized by the Ernst angle $\theta_{E}$ :

$$
\cos \theta_{E}=\frac{e^{-\gamma}+e^{-\Gamma}}{1+e^{-\Gamma-\gamma}}
$$

The dissipation effect is neglected during the control time. The coordinates of the corresponding measurement point $M$ are:

$$
z_{m}^{(E)}=\frac{1}{1+e^{\gamma}} ; y_{m}^{(E)}=\frac{e^{\Gamma}}{1+e^{\gamma}} \sqrt{\frac{e^{2 \gamma}-1}{e^{2 \Gamma}-1}} .
$$

The figure of merit $F_{E}$ is then given by $F_{E}=y_{m}^{(E)}$ since the detection time $T_{d}$ is set to 1 and the control time $T_{c}$ is zero for an ideal $\delta$-pulse. 
We start the analysis of the optimal control algorithm by a general study of the maximum SNR that can be achieved for short control durations. As can be seen in Fig. 2, we first verify that the algorithm converges to the Ernst angle solution when the control duration goes to 0 . We observe that the convergence is almost linear as a function of $T_{c}$. Figure 3 represents the different positions of the steady state and the measurement point during the optimization process. Figure 3 clearly shows that the two points converge very quickly towards the points of the Ernst angle solution.

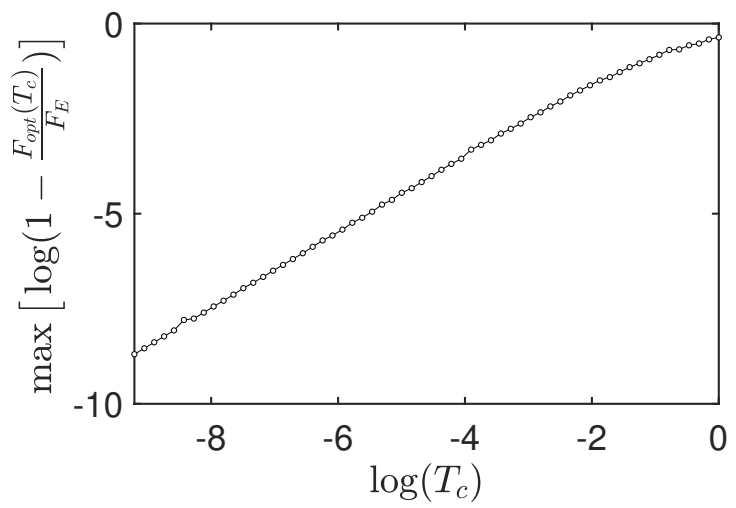

FIG. 2: (Color online) Evolution of the figure of merit $F_{\text {opt }}\left(T_{c}\right)$ for different control times $T_{c}$ (open circles). The relaxation parameters are set to $\Gamma=4$ and $\gamma=2$. The maximum is taken over 20 realizations of the algorithm for each time $T_{c}$ with different random trial fields. Dimensionless units are used.

In a second series of numerical tests, we consider a range of times $T_{c}$ in the interval $\left[10^{-4}, 7.3 \times 10^{-4}\right]$. Several optimizations are performed for increasing control times, in which the previous optimal field is used as a guess field to initialize the next optimization. A coarse and a fine discretizations of the time interval $\left[10^{-4}, 7.3 \times 10^{-4}\right]$ are used, with 4 and 13 points, respectively. The results of the optimization process are given in Fig. 4. A smooth evolution is observed in the case of a fine discretization, while an abrupt change occurs for the coarse one. The different control mechanisms can be described from the analysis of the uniform norm of the optimal field, $\|\omega\|_{\infty}=\max _{t \in\left[0, T_{c}\right]}\left|\omega_{x}(t)\right|$. Figure 4 shows that this norm is almost constant for the coarse case, which leads to different trajectories as can be seen in Fig. 5 (See also the movies in the Supplemental Material [59]). For times longer than $3 \times 10^{-4}$, the steady state and the measurement points change and the system follows more complex trajectories. For the fine case, the figure of merit changes very little from one control time to the next. For all the possible values of $T_{c}$, we observe that the optimal solution is very similar to the one for $T_{c}=10^{-4}$. The uniform norm of the control field decreases with $T_{c}$ so that the area of the field remains approximately constant.

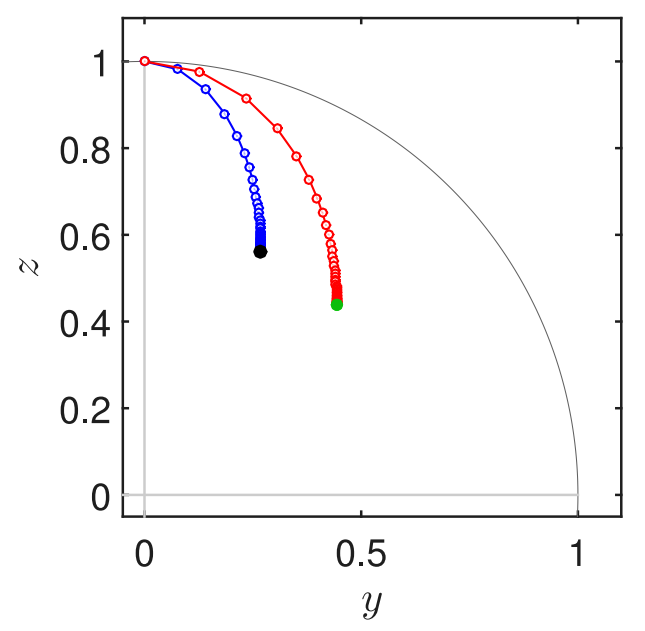

FIG. 3: (Color online) Evolution of the position of the steady state $S$ (blue or dark gray) and the measurement point $M$ (red or light gray) during the optimization process (the points of the first 25 iterations of the algorithm are plotted). Since the guess field is a zero control, the initial position of $S$ and $M$ corresponds to the north pole of Bloch sphere of coordinates $(0,1)$. The $S$ and the $M$ points for the Ernst angle solution are depicted respectively in black and in green. The control time $T_{c}$ is set to $10^{-7}$ and the relaxation parameters to $\Gamma=4$ and $\gamma=2$. Dimensionless units are used.

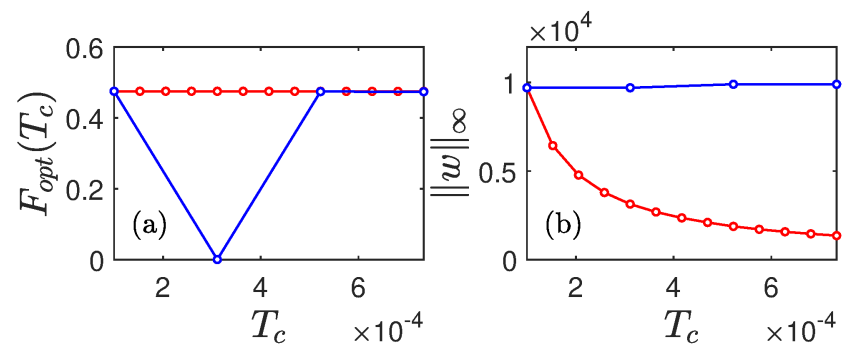

FIG. 4: (Color online) Evolution of the figure of merit $F_{o p t}$ (panel (a)) and of the corresponding uniform norm of $\omega(t)$ (panel (b)) as a function of $T_{c}$ (open circles). The coarse and the fine time discretizations are plotted respectively in blue (dark gray) and in red (light gray) lines. The relaxation parameters $\Gamma$ and $\gamma$ are set respectively to 4 and 2. Dimensionless units are used.

\section{B. Extension to a spin ensemble with different offsets}

We investigate in this section the efficiency of the numerical algorithm for optimizing an inhomogeneous spin ensemble. As a first example, we consider the control of 4 spins with different offsets. The optimization algorithm has been used for a specific control time $T_{c}=5.22 \times 10^{-4}$. The optimal trajectories are plotted in Fig. 6 for the four offsets. The set of steady state and measurement points are also represented for $\omega \in[0,10]$. The two sets of points are distributed along two circles of the Bloch ball. The figure of merit is given in Fig. 7 where the result for a homogeneous ensemble is also indicated. Note the ro- 


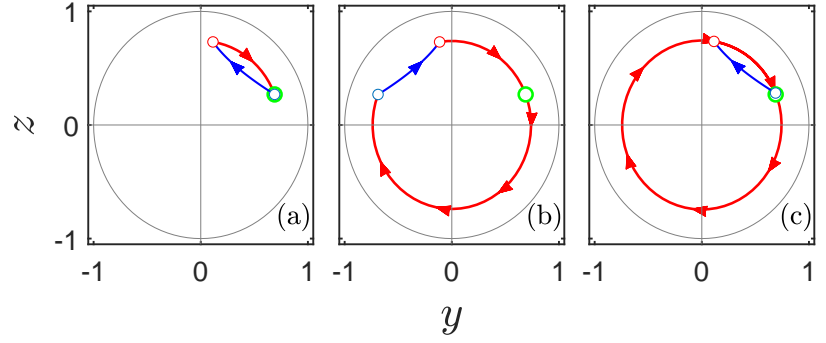

FIG. 5: (Color online) Optimal trajectories for $T_{c}=10^{-4}$ (panel (a)), $4.16 \times 10^{-4}$ (panel (b)) and $7.33 \times 10^{-4}$ (panel (c)) corresponding to the coarse discretization of Fig. 4 . The open blue (dark gray) and red (light gray) circles represent respectively the steady state and measurement points. The controlled trajectory is plotted in solid red (light gray) line, the free relaxation in solid blue (dark gray) line. The measurement point of the Ernst angle solution corresponds to the green open circle. Dimensionless units are used.

bustness of $F_{\text {opt }}$ against variation of the offset $\omega$ in the interval $[0,10]$. This observation is not valid for larger offsets.

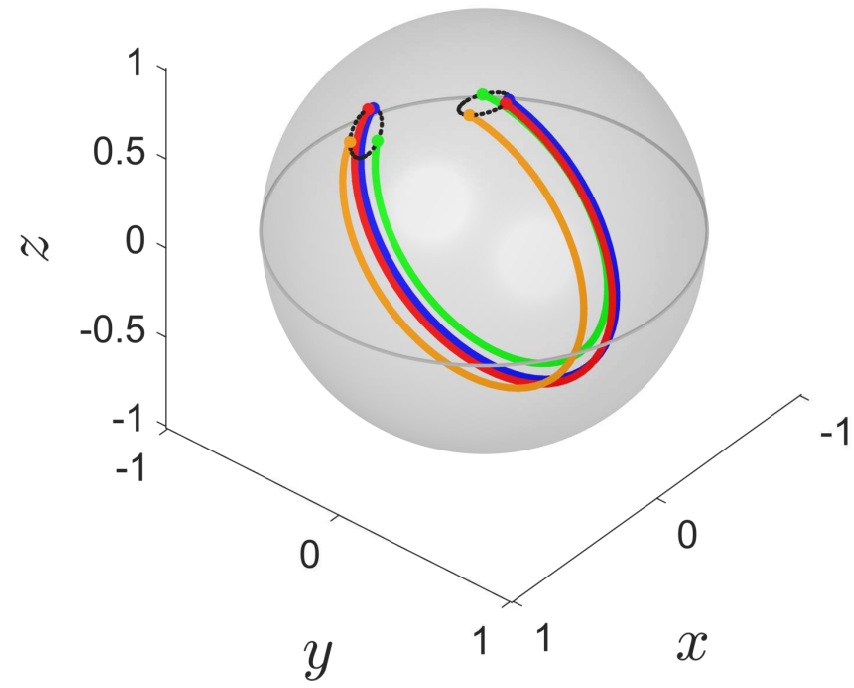

FIG. 6: (Color online) Plot of the controlled trajectories for different offsets in the $(x, y, z)$ - space. The parameters are set to $T_{c}=5.22 \times 10^{-4}, \Gamma=1.8, \gamma=1$ and the four offsets are: $\omega_{1}=3.3333, \omega_{2}=5.5555, \omega_{3}=7.7778$ and $\omega_{4}=10$. The dashed lines indicate the positions of the steady states and measurement points in the interval $[0,10]$. Dimensionless parameters are used.

\section{CONCLUSION}

We have proposed in this study a numerical optimization algorithm for quantum systems with a periodic time evolution. The difficulty and the originality of the procedure rely on the fact that the initial and target states of the dynamics are not known but have to be optimized

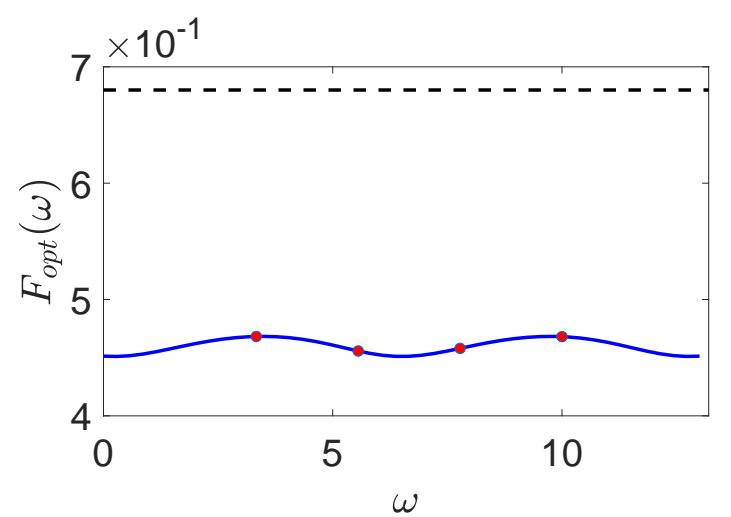

FIG. 7: (Color online) Evolution of the figure of merit $F_{\text {opt }}$ as a function of the offset $\omega$. The red dots represent the four offsets used in the numerical optimization. The same dimensionless parameters as in Fig. 6 are used. The horizontal dashed line displays the figure of merit for a homogeneous spin ensemble with the same relaxation parameters $\Gamma$ and $\gamma$.

together with the control field. The algorithm is built on a standard framework, except for the computation of the initial state and adjoint state of the system. A time discretization scheme of the algorithm is presented. It has the advantage of simplicity and general applicability. As an illustrative example, we have considered the maximization of the SNR for an ensemble of spin 1/2 particles. We have shown that the algorithm converges to the Ernst angle solution with a very high efficiency in the limit of a control duration going to 0 . This analysis leads also to important insights into the design of optimal pulses. According to the used guess field, we have observed that the algorithm converges towards different fields associated with different steady states and measurement points. The different trajectories in the Bloch ball can be geometrically characterized. Some preliminary computations have also be done in the case of an inhomogeneous spin ensemble with different offsets. The results of this paper can be viewed as an important step forward in the development of numerical optimal algorithms in quantum dynamics. In the case of an inhomogeneous spin ensemble, only four spins have been considered in this paper and a first improvement would be the speed-up of the algorithm in order to reduce the computational time, e.g. by using parallelization techniques [60]. Another open question is the mathematical and numerical description of the transient regime, only the permanent periodic dynamics have been investigated in this work. Some results have been established in this direction for the Ernst angle solution, but it will be interesting to generalize this analysis to optimal fields of non-zero duration [49-51]. 


\section{APPENDIX A: THE ERNST ANGLE SOLUTION}

The goal of this paragraph is to recall the main features of the Ernst angle solution. We consider the case of a spin $1 / 2$ particle subjected to a control field along the $x$-direction. We denote respectively by $S\left(y_{s}, z_{s}\right)$ and $M\left(y_{m}, z_{m}\right)$ the steady state and the measurement point of the control process. The $S$ and the $M$ points are connected by a field-free evolution:

$$
\left\{\begin{array}{l}
y_{s}=y_{m} e^{-\Gamma} \\
z_{s}=1-e^{-\gamma}+z_{m} e^{-\gamma}
\end{array}\right.
$$

In this limiting case, the pulse sequence is reduced to an ideal $\delta$-pulse, the radial coordinates $r_{s}$ and $r_{m}$ are therefore the same. We deduce that:

$$
y_{m}^{2} e^{-2 \Gamma}+\left(1-e^{-\gamma}+z_{m} e^{-\gamma}\right)^{2}=y_{m}^{2}+z_{m}^{2} .
$$

The figure of merit $F$ is here given by $y_{m}$. A necessary condition to maximize $F$ is $\frac{d y_{m}}{d z_{m}}=0$. We obtain the following relation:

$$
z_{m}-\left(1-e^{-\gamma}+z_{m} e^{-\gamma}\right) e^{-\gamma}=0,
$$

leading to:

$$
z_{m}=\frac{1-e^{-\gamma}}{e^{\gamma}-e^{-\gamma}}
$$

which can be transformed into:

$$
z_{m}=\frac{1}{1+e^{\gamma}} .
$$

It is then straightforward to show that:

$$
y_{m}=\frac{e^{\Gamma}}{1+e^{\gamma}} \sqrt{\frac{e^{2 \gamma}-1}{e^{2 \Gamma}-1}} .
$$

We then deduce that the Ernst angle which characterizes the $\delta$-pulse can be expressed as:

$$
\cos (\theta)=\cos \left(\theta_{m}-\theta_{s}\right)=\frac{z_{m} z_{s}+y_{m} y_{s}}{y_{m}^{2}+z_{m}^{2}} .
$$

Using the preceding formulas, we obtain:

$$
y_{m}^{2}+z_{m}^{2}=\frac{1}{\left(1+e^{\gamma}\right)^{2}} \frac{1-e^{2(\Gamma+\gamma)}}{1-e^{2 \Gamma}},
$$

and

$$
y_{m} y_{s}+z_{m} z_{s}=\frac{e^{\Gamma}}{\left(1+e^{\gamma}\right)^{2}} \frac{e^{2 \gamma}-1}{e^{2 \Gamma}-1}+\frac{e^{\gamma}}{\left(1+e^{\gamma}\right)^{2}}
$$

We arrive at:

$$
\cos \theta=\frac{e^{\gamma}\left(1-e^{2 \Gamma}\right)+e^{\Gamma}\left(1-e^{2 \gamma}\right)}{1-e^{2(\Gamma+\gamma)}},
$$

which can be simplified into:

$$
\cos \theta=\frac{e^{-\Gamma}-e^{\Gamma}+e^{-\gamma}-e^{\gamma}}{e^{-(\gamma+\Gamma)}-e^{\gamma+\Gamma}}
$$

and into the final formula:

$$
\cos \theta=\frac{e^{-\gamma}+e^{-\Gamma}}{1+e^{-\Gamma-\gamma}}
$$

which is the well-known formulation of the Ernst angle solution.

\section{ACKNOWLEDGMENT}

S.J. Glaser acknowledges support from the DFG (GI 203/7-1), SFB 631 and the BMBF FKZ 01EZ114 project. D. Sugny and S. J. Glaser acknowledge support from the ANR-DFG research program Explosys (ANR-14-CE350013-01). The work of D. Sugny has been done with the support of the Technische Universität München Institute for Advanced Study, funded by the German Excellence Initiative and the European Union Seventh Framework Programme under grant agreement 291763. J. Salomon is funded by ANR Ciné-Para (ANR-15-CE23-0019). This project has received funding from the European Union's Horizon 2020 research and innovation programme under the Marie-Sklodowska-Curie grant agreement No 765267.
[1] L. Pontryagin, Mathematical theory of optimal processes (Mir, Moscow, 1974)

[2] A. Bryson and Y.-C. Ho, Applied Optimal Control (Hemisphere, Washington, DC, 1975)

[3] B. Bonnard and D. Sugny, Optimal control in space and quantum dynamics (AIMS applied Math. Vol. 5, 2012)

[4] V. Jurdjevic, Geometric control theory (Cambridge University Press, Cambridge, 1996)

[5] P. Brumer and M. Shapiro, Principles and Applications of the quantum control of molecular processes (Wiley Interscience, 2003)
[6] S. A. Rice and M. Zhao, Optimal control of molecular dynamics (John Wiley and sons, 2000)

[7] D. D'Alessandro, Introduction to quantum control and dynamics (Chapman and Hall, Boca Raton, 2008)

[8] N. Khaneja, R. Brockett and S. J. Glaser, Phys. Rev. A 63, 032308 (2001)

[9] M. Lapert, Y. Zhang, M. Braun, S. J. Glaser and D. Sugny, Phys. Rev. Lett. 104 (2010) 083001.

[10] D. Sugny and C. Kontz, Phys. Rev. A 77, 063420 (2008)

[11] A. Garon, S. J. Glaser and D. Sugny, Phys. Rev. A 88, $043422(2013)$ 
[12] S. J. Glaser, U. Boscain, T. Calarco, C. Koch, W. Kockenberger, R. Kosloff, I. Kuprov, B. Luy, S. Schirmer, T. Schulte-Herbrüggen, D. Sugny and F. Wilhelm, Eur. Phys. J. D 69, 279 (2015)

[13] C. Brif, R. Chakrabarti and H. Rabitz, New J. Phys. 12 , 075008 (2010)

[14] C. Altafini and F. Ticozzi, IEEE Trans. Automat. Control 57, 1898 (2012)

[15] D. Dong and I. A. Petersen, IET Control Theory A. 4, $2651(2010)$

[16] N. Khaneja, T. Reiss, C. Kehlet, T. Schulte-Herbrüggen and S. J. Glaser, J. Magn. Reson. 172, 296 (2005)

[17] D. M. Reich, M. Ndong and C. P. Koch, J. Chem. Phys. 136, 104103 (2012)

[18] Y. Maday and G. Turinici, J. Chem. Phys. 118, 8191 (2003)

[19] W. Zhu, J. Botina, and H. Rabitz, J. Chem. Phys. 108, 1953 (1998).

[20] R. Kosloff, S. A. Rice, P. Gaspard, S. Tersigni, and D. Tannor, Chem. Phys. 139, 201 (1989).

[21] J. Somloi, V. A. Kazakovski, and D. J. Tannor, Chem. Phys. 172, 85 (1993)

[22] T. E. Skinner, T. O. Reiss, B. Luy, N. Khaneja, and S. J. Glaser, J. Magn. Reson. 172, 17 (2005)

[23] K. Kobzar, T. E. Skinner, N. Khaneja, S. J. Glaser, and B. Luy, J. Magn. Reson. 170, 236 (2004)

[24] K. Kobzar, T. E. Skinner, N. Khaneja, S. J. Glaser, and B. Luy, J. Magn. Reson. 194, 58 (2008)

[25] S. Conolly, D. Nishimura and A. Macovski, IEEE Trans. Med. Imaging 5, 106 (1986)

[26] M. Lapert, Y. Zhang, M. Janich, S. J. Glaser and D. Sugny, Sci. Rep. 2, 589 (2012)

[27] H. Liu and G.B. Matson, Magn. Reson. Med. 66, 1254 (2011)

[28] M. S. Vinding, I. I. Maximov, Z. Tosner and N. C. Nielsen, J. Chem. Phys. 137, 5 (2012)

[29] I. I. Maximov, M. S. Vinding, H. Desmond, N. C. Nielsen and N.J. Shah, J. Magn. Reson. 254, 110 (2015)

[30] D. Xu, K. F. King, Y. Zhu, G. C. McKinnon and Z.-P. Liang, Magn. Reson. Med. 59, 547 (2008)

[31] A. Massire, M. A. Cloos, A. Vignaud, D. L. Bihan, A. Amadon and N. Boulant, J. Magn. Reson. 230, 76 (2013)

[32] B. Bonnard, O. Cots, S. Glaser, M. Lapert, D. Sugny and Y. Zhang, IEEE Trans. Autom. Control 57, 1957 (2012)

[33] A. Sbrizzi, H. Hoogduin, J. V. Hajnal, C. A. van den Berg, P. R. Luijten, S. J. Malik, Magn. Reson. Med. 77, 361 (2017)

[34] E. Van Reeth, H. Ratiney, M. Tesch, D. Grenier, O. Beuf, S. J. Glaser, D. Sugny, J. Magn. Reson. 279, 39 (2017)

[35] J. Werschnik and E. K. U. Gross, J. Phys. B 40, R175 (2007)
[36] Y. Zhang, M. Lapert, M. Braun, D. Sugny, and S. J. Glaser, J. Chem. Phys. 134, 054103 (2011)

[37] D. Sugny, S. Vranckx, M. Ndong, N. Vaeck, O. Atabek and M. Desouter-Lecomte, Phys. Rev. A 90, 053404 (2014)

[38] P. E. Spindler, Y. Zhang, B. Endeward, N. Gershenzon, T. E. Skinner, S. J. Glaser, and T. F. Prisner, J. Magn. Reson. 218, 49 (2012)

[39] T. E. Skinner, T. O. Reiss, B. Luy, N. Khaneja, and S. J. Glaser, J. Magn. Reson. 167, 68 (2004)

[40] G. Dridi, M. Lapert, J. Salomon, S. J. Glaser and D. Sugny, Phys. Rev. A 92, 043417 (2015)

[41] M. Lapert, G. Ferrini and D. Sugny, Phys. Rev. A 85, 023611 (2012)

[42] E. G. Gilbert, SIAM J. Control and Optim. 15, 717 (1977)

[43] F. Colonius, Optimal periodic control (Springer-Verlag, Berlin, 1988)

[44] T. Bayen, F. Mairet, P. Martinon, and M. Sebbah, Optimal Control Appl. Methods, 36, 750 (2015)

[45] M. A. Bernstein, K. F. King and X. J. Zhou, Handbook of MRI pulse sequences (Elsevier, London, 2004)

[46] R. R. Ernst, Principles of Nuclear Magnetic Resonance in one and two dimensions (International series of monographs on chemistry, Oxford, 1990)

[47] M. H. Levitt, Spin dynamics: Basics of Nuclear Magnetic Resonance (Wiley, New York, 2008)

[48] R. R. Ernst and W. A. Anderson, Rev. Sci. Instrum. 37, 93 (1966)

[49] K. Scheffler, Magn. Reson. Med. 49, 781 (2003)

[50] V. S. Deshpande, Y.-C. Chung, Q. Zhang, S. M. Shea, D. Li, Magn. Reson. Med. 49, 151 (2003)

[51] B. A. Hargreaves, S. S. Vasanawala, J. M. Pauly and D. G. Nishimura, Magn. Reson. Med. 46, 149 (2001)

[52] C. Ganter, Magn. Reson. Med. 52, 368 (2004)

[53] C. Ganter, Magn. Reson. Med. 62, 149 (2009)

[54] P. W. Worters and B. A. Hargreaves, Magn. Reson. Med. 64, 1404 (2010)

[55] O. Bieri and K. Scheffler, J. Magn. Reson. Imaging 38, 2 (2013)

[56] K. Scheffler and S. Lehnhardt, Eur. Radiol. 13, 2409 (2003)

[57] M. Lapert, E. Assémat, S. J. Glaser and D. Sugny, Phys. Rev. A 90, 023411 (2014)

[58] M. Lapert, E. Assémat, S. J. Glaser and D. Sugny, J. Chem. Phys. 142, 044202 (2015)

[59] See Supplemental Material for a video about the optimal trajectories of the spins in the three cases of Fig. 5.

[60] M. K. Riahi, J. Salomon, S. J. Glaser and D. Sugny, Phys. Rev. A 93, 043410 (2016) 